\title{
Distinct molecular signature of phospholamban p.Arg14del arrhythmogenic cardiomyopathy
}

Wouter P. te Rijdt, $\mathrm{MD}^{1-3}$, Angeliki Asimaki, PhD ${ }^{4}$, Jan D.H. Jongbloed, MD, PhD ${ }^{1}$, Edgar T. Hoorntje, $M^{1}$, Elisabetta Lazzarini ${ }^{5}$, Paul A. van der Zwaag, MD, $\mathrm{PhD}^{1}$, Rudolf A. de Boer, MD, PhD ${ }^{3}$, J. Peter van Tintelen, MD, $\mathrm{PhD}^{6,7}$, Jeffrey E. Saffitz, MD, $\mathrm{PhD}^{8}$, Maarten P. van den Berg, MD, PhD ${ }^{3}$, Albert J.H. Suurmeijer, MD, $\mathrm{PhD}^{9}$

${ }^{1}$ University of Groningen, University Medical Center Groningen, Department of Genetics, Groningen, the Netherlands. ${ }^{2}$ Netherlands Heart Institute, Utrecht, the Netherlands. ${ }^{3}$ University of Groningen, University Medical Center Groningen, Department of Clinical and Experimental Cardiology, Groningen, the Netherlands. ${ }^{4}$ Cardiology Clinical Academic Group, St. George's University of London, Cranmer Terrace, London, United Kingdom. ${ }^{5}$ Departments of Cardiac, Thoracic, and Vascular Sciences, University of Padua, Padua, Italy. ${ }^{6}$ Department of Clinical Genetics, Amsterdam Cardiovascular Sciences, Academic Medical Center, University of Amsterdam, Amsterdam, the Netherlands ${ }^{7}$ Durrer Center for Cardiovascular Research, Netherlands Heart Institute, Utrecht, the Netherlands ${ }^{8}$ Department of Pathology, Beth Israel Deaconess Medical Center \& Harvard Medical School, Boston, MA, USA. ${ }^{9}$ University of Groningen, University Medical Center Groningen, Department of Pathology, Groningen, the Netherlands.

Brief title: Distinct features of Phospholamban p.Arg14del cardiomyopathy

Total word count: $3347 \quad$ Figures: 2 Tables: 1

\author{
Corresponding author: \\ Wouter Paul te Rijdt, M.D. \\ Department of Clinical Genetics, Cardiology and Pathology \\ University Medical Center Groningen \\ PO Box 30001 \\ 9700 RB Groningen, the Netherlands \\ Phone: +31 503617534 \\ Fax: +31503615525 \\ E-mail: w.p.te.rijdt@umcg.nl
}




\section{Abstract}

Phospholamban (PLN) p.Arg14del cardiomyopathy is characterized by a distinctly arrhythmogenic biventricular phenotype that can be predominantly left ventricular, right ventricular, or both. Our aim was to further elucidate distinct features of this cardiomyopathy with respect to the distribution of desmosomal proteins observed by immunofluorescence in comparison to desmosomal arrhythmogenic cardiomyopathy and co-existent genetic variants. We studied 8 explanted heart specimens from PLN p.Arg14del mutation carriers. Macro- and microscopic examination revealed biventricular presence of fibrofatty replacement and interstitial fibrosis. Five out of 8 (63\%) of patients met consensus criteria for both arrhythmogenic right ventricular cardiomyopathy (ARVC) and dilated cardiomyopathy (DCM). In four cases, targeted NGS revealed one additional pathogenic variant and six variants of unknown significance. Immunofluorescence showed diminished junction plakoglobin signal intensity at the intercalated disks in 4/6 (67\%) cases fulfilling ARVC criteria but normal intensity in both cases fulfilling only DCM criteria. Notably, the 4 cases with diminished junction plakoglobin were also those where an additional gene variant was detected. Immunofluorescence for two proteins recently investigated in desmosomal ACM, synapse-associated protein 97 and glycogen synthase kinase-3 beta, showed a distinct distributional pattern in comparison to desmosomal ACM. In 7/8 (88\%) cases we observed both a strong synapse-associated protein 97 signal at the sarcomeres and no glycogen synthase kinase-3 beta translocation to the intercalated discs. Phospholamban p.Arg14del cardiomyopathy is characterized by a distinct molecular signature compared to desmosomal ACM, specifically a different desmosomal protein distribution. This study substantiates the idea that additional genetic variants play a role in the phenotypical heterogeneity.

\section{Key words}

phospholamban; arrhythmogenic cardiomyopathy; dilated cardiomyopathy; immunofluorescence; next-generation sequencing 


$\begin{array}{ll}\text { Abbreviations } & \\ \text { ACM } & \text { Arrhythmogenic cardiomyopathy } \\ \text { ARVC } & \text { Arrhythmogenic right ventricular cardiomyopathy } \\ \text { DCM } & \text { Dilated cardiomyopathy } \\ \text { IHC } & \text { Immunofluorescence } \\ \text { LV } & \text { Left Ventricle/ventricular } \\ \text { MAGUK } & \text { Membrane-associated guanylate kinase } \\ \text { NGS } & \text { Next-generation sequencing } \\ \text { PLN } & \text { Phospholamban } \\ \text { RV } & \text { Right ventricle/ventricular } \\ \text { SERCA } & \text { Sarcoplasmic reticulum Ca }{ }^{2+} \text {-ATPase } \\ \text { SNP } & \text { Single nucleotide polymorphism } \\ \text { VUS } & \text { Variant of unknown significance }\end{array}$




\section{Introduction}

The most common forms of cardiomyopathy, classified according to their phenotypic expression, are hypertrophic, dilated (DCM), and arrhythmogenic cardiomyopathy (ACM), but overlapping phenotypes are well recognized. ${ }^{1} \mathrm{ACM}$ encompasses a broad spectrum of disease that includes the classical right-dominant form (arrhythmogenic right ventricular cardiomyopathy; ARVC), predominant left-sided involvement (also referred to as left-dominant arrhythmogenic cardiomyopathy; LDAC) and biventricular subtypes. Ventricular arrhythmias and an increased risk of sudden cardiac death are characteristic of all subtypes, particularly in the young and those active in sports. ${ }^{2}$ In ACM, the electrical instability exceeds the degree of LV dysfunction as compared to DCM. Most ACM-associated pathogenic variants are found in desmosomal genes, but non-desmosomal pathogenic variants have more recently also been identified. ${ }^{3,4}$

Following the identification of the non-desmosomal p.Arg14del (c.40_42delAGA) mutation in the PLN gene in a large subset of DCM and ACM patients, it has been shown that mutation carriers are at increased risk of malignant ventricular arrhythmias and severe heart failure and have an impaired prognosis from late adolescence. ${ }^{5-7}$ Histopathologically, the disease has been demonstrated to be characterized by biventricular fibrofatty replacement and interstitial fibrosis. Furthermore, PLN-containing aggregates that are concentrated in dense perinuclear aggresomes in cardiomyocytes were observed..$^{8-10}$ Patients with PLN p.Arg14del cardiomyopathy may fulfil international DCM criteria $^{11}$ and/or ARVC revised task force criteria. ${ }^{12}$ In a previous study, immunofluorescence analysis of biopsy samples revealed depressed or absent junction plakoglobin signal intensity at intercalated disks in the majority (71\%) of cases fulfilling ARVC criteria, but this was seen in only one out of nine (11\%) DCM cases. ${ }^{5}$

In desmosomal ACM, recently synapse-associated protein 97 and glycogen synthase kinase-3 beta were studied. Synapse-associated protein 97 is a membrane-associated guanylate kinase (MAGUK) involved in trafficking sodium and potassium channel subunits to the cell surface, which helps maintain normal cardiac ventricular resting membrane action potential. This PDZ-domain 
containing protein was found to be consistently reduced in the ventricular myocardium of desmosomal ACM-patients, an effect that is independent of the specific causal mutation. ${ }^{13}$ Glycogen synthase kinase-3 beta is a ubiquitously expressed serine/threonine kinase that remains active in the resting state and is inactivated when stimulated by, e.g., the Wnt pathway. Asimaki et al. ${ }^{13}$ identified a small molecule (SB2) as an inhibitor of glycogen synthase kinase-3 beta because it rescued the ACM phenotype in a zebrafish model, thereby increasing canonical Wnt signaling. All the desmosomal human ACM cases investigated (20/20) showed an abnormal glycogen synthase kinase-3 beta immunoreactive signal at the intercalated disks. ${ }^{14}$ Expression of these proteins has not been studied in PLN p.Arg14del cardiomyopathy.

Further elucidation of the distribution of desmosomal proteins and co-existent genetic variants in PLN p.Arg14del cardiomyopathy may help us to better understand the observed phenotypic heterogeneity and improve diagnosic- and treatment options. In order to study this, histologic examination, immunofluorescence, next-generation sequencing (NGS) for additional cardiomyopathy-associated genetic variants and clinical evaluation according to consensus criteria for $\mathrm{DCM}^{11}$ and $A R V C^{12}$ were performed in PLN p.Arg14del cardiomyopathy heart transplant cases. 


\section{Materials and Methods}

\subsection{Clinical and pathological examination}

We evaluated eight explanted hearts from index patients who were heterozygous carriers of the PLN p.Arg14del mutation from the Department of Pathology of the University Medical Center Groningen in the Netherlands. Information on gross examination of the hearts was available for all cases because they were evaluated during transplant procedures. Formalin-fixed and paraffin-embedded samples from, at minimum, the anterior, lateral and posterior wall of the left ventricle (LV), as well as the right ventricular (RV) wall, were available for all cases. Tissue sections $(4 \mu \mathrm{m})$ were stained with hematoxylin and eosin as well as Masson's trichrome. Tissue sections from the LV and RV were evaluated under light microscopy by recording the presence of myocyte hypertrophy and interstitial fibrosis and the presence of fibrofatty replacement. The presence of interstitial fibrosis in the LV wall was evaluated either as not present, present in the subepicardial half of the myocardial wall or transmurally present. The presence of fibrofatty replacement was evaluated by naked eye examination of Masson's-trichrome-stained RV wall sections placed on a sheet of white paper.

Clinical data of the eight Dutch index patients was collected from the PHORECAST registry (PHOspholamban RElated CArdiomyopathy STudy; http://www.phorecast.nl). The clinical criteria proposed by Mestroni et al. ${ }^{11}$ were used to diagnose DCM and the revised task force criteria to diagnose ARVC. ${ }^{12}$

\subsection{Ethics Statement}

The PHORECAST registry complies with the 'Code of conduct for medical research' drawn up by the Dutch Federation of Biomedical Scientific Societies (FMWV). The laws in force in the Personal Data Protection Act (WBP) and the Medical Treatment Contracts Act (WGBO) are incorporated in this code of conduct. This study met the criteria of the code of conduct for responsible use of human tissue that is used in the Netherlands (Dutch federation of biomedical scientific societies;

http://www.federa.org). The study conformed to the principles of the Helsinki Declaration; all 
material was originally obtained for diagnostic procedures and the tissue samples were de-identified before inclusion in the study.

\subsection{Gene variant analysis by targeted NGS}

Targeted NGS using a previously validated NGS method ${ }^{15}$ was performed for all eight PLN p.Arg14del mutation carriers to search for additional variants in 61 known cardiomyopathy-associated genes. Details of sample preparation, sequencing, data processing, and variant filtering and classification are summarized in the Supplementary File. American College of Medical Genetics and Genomics and Association for Molecular Pathology criteria were used for variant classification. ${ }^{16}$ Only variants of uncertain significance (VUS), likely pathogenic variants (LPV), or pathogenic variants (PV) were reported.

\subsection{Immunofluorescence}

Blinded patient samples were subjected to fluorescent immunofluorescence (IHC) as previously described. ${ }^{6,17,18}$ Tissue samples from age-matched individuals with no clinical or pathological evidence of heart disease were subjected to the same protocol and used as negative controls $(n=5)$. Briefly, $4 \mu \mathrm{m}$-thick formalin-fixed, paraffin-embedded tissue sections were deparaffinised, dehydrated, rehydrated and heated in citrate buffer $(10 \mathrm{mmol} / \mathrm{l}, \mathrm{pH} 6.0)$ to enhance specific immunofluorescence. After being cooled to room temperature, the tissue sections were simultaneously permeabilized and blocked by incubating them in phosphate-buffered saline containing $1 \%$ Triton $\mathrm{X}-100,3 \%$ normal goat serum and $1 \%$ bovine serum albumin. The sections were then incubated, first with a primary antibody and then with indocarbocyanine-conjugated goat antimouse or anti-rabbit rabbit IgG. The primary antibodies were the same as those used in previous studies: mouse monoclonal anti-N-cadherin (SIGMA), rabbit polyclonal anti-gap junction protein (SIGMA), mouse monoclonal anti-junction plakoglobin (SIGMA), mouse monoclonal anti-desmoplakin 
(Fitzgerald), rabbit polyclonal anti-synapse-associated protein 97 (Santa Cruz Biotechnology) and rabbit polyclonal anti- glycogen synthase kinase-3 beta (Cell Signaling). ${ }^{17,18}$

\section{Results}

\subsection{Patient characteristics, clinical data and histopathology}

The eight explant heart specimens were from three males and five females with end-stage heart failure (mean[ $[ \pm S D]$ age $53 \pm 11$ years; Table 1 ). Macro- and microscopic examination of the explanted heart specimens revealed features of both ARVC and DCM: biventricular presence of fibrofatty replacement that was most prominent in the RV wall and interstitial fibrosis that was most prominent in the subepicardial LV wall (Figure 1: case 5 as an typical example), as previously observed. ${ }^{8-10}$ Five out of $8(63 \%)$ patients met consensus criteria for both ARVC and DCM. Two out of $8(25 \%)$ met consensus criteria for DCM only and one case for ARVC only (Table 1 ).

\subsection{Variant analysis}

Targeted NGS confirmed the pathogenic p.Arg14del mutation in PLN in all cases. In addition, one PV, no LPVs and 6 VUSs were found in cardiomyopathy-associated genes in four cases: emerin (EMD; one VUS), titin (TTN; three VUS, including two in one patient), myosin binding protein C (MYBPC3; one PV), troponin T (TNNT2; one VUS) and plakophilin2 (PKP2; one VUS). Details of the genetic variants are shown in Table 1.

\subsection{Immunofluorescence}

All cases showed a control signal similar in intensity and distribution for gap junction protein (the major cardiac gap junction protein normally located in the intercalated discs), desmoplakin, and $\mathrm{N}$ Cadherin (used as a tissue quality control). Junction plakoglobin signal intensity was depressed or absent at the intercalated disks in the majority $(4 / 6 ; 67 \%)$ of cases fulfilling ARVC criteria, but signal intensity was normal at the intercalated disks in the two cases only fulfilling DCM criteria (Table 1). 
Synapse-associated protein 97 immunofluorescence showed a strong signal at the sarcomeres in $7 / 8(88 \%)$ cases, though the signal was gone from the intercalated disks (Table 1) as was observed previously in myocardial samples from patients with end-stage ischemic, dilated, or hypertrophic cardiomyopathy. ${ }^{13}$ Case 1 , however, showed overall diminished signal intensity for Synapse-associated protein 97 (Figure 2) as previously shown in hearts from ACM patients with desmosomal gene mutations. ${ }^{13}$

Immunofluorescence for glycogen synthase kinase-3 beta revealed that this protein retained its cytoplasmic distribution and did not translocate to the intercalated disks in the same $7 / 8(88 \%)$ cases that showed a strong signal for synapse-associated protein 97 at the sarcomeres (Table 1). Case 1 was the only case that showed junctional redistribution for glycogen synthase kinase-3 beta (Figure 2), which is in contrast to what was seen in a desmosomal ACM cohort in which all 20 cases showed glycogen synthase kinase-3 beta junctional redistribution. ${ }^{14}$ 


\section{Discussion}

In recent years the idea has emerged that pathogenic variants within a single specific cardiomyopathy-related gene may lead to several cardiomyopathy subtypes, i.e. phenotypic heterogeneity. A typical example of this phenomenon occurs in PLN p.Arg14del-related cardiomyopathy, where many symptomatic PLN p.Arg14del carriers have overlapping phenotypes and may fulfil international DCM criteria ${ }^{11}$ and/or ARVC revised task force criteria. ${ }^{12} \mathrm{LV}$ involvement is typical in PLN p.Arg14del cardiomyopathy. ${ }^{3,10,19}$

In line with previous findings in PLN p.Arg14del cardiomyopathy, we observed a biventricular phenotype with features of both ARVC and DCM. This challenges the strict distinction made between ARVC and DCM as two separate entities. The hearts showed biventricular presence of fibrofatty replacement and interstitial fibrosis, in line with our previous pathological ${ }^{8-10}$ and cardiac magnetic resonance imaging findings. ${ }^{20}$ Sepehrkhouy et al. recently showed this fibrosis pattern to be distinctive for PLN p.Arg14del cardiomyopathy in comparison with other hereditary cardiomyopathies. ${ }^{10}$ Clinically, the majority of patients (63\%) in our cohort fulfilled criteria for both ARVC and DCM. Task force criteria are only available for ARVC ${ }^{12}$, but are not sufficient for LDAC. ${ }^{21}$ Phospholamban p.Arg14del cardiomyopathy is a typical example of a biventricular ACM phenotype.

Differential abnormalities in the distribution and expression of desmosomal and gap junction proteins have been reported in cardiac tissue of patients with ACM and DCM. ${ }^{17}$ Previous immunofluorescence analysis of biopsy samples revealed depressed or absent junction plakoglobin signal intensity at intercalated disks in the majority (71\%) of cases fulfilling ARVC criteria, but this was seen in only one out of nine (11\%) DCM cases. ${ }^{5}$ Junction plakoglobin redistribution thus seemed to track with phenotype rather than genotype. Interestingly, López-Ayala et al. ${ }^{22}$ also saw no junction plakoglobin redistribution in a similar LDAC phenotype caused by a desmoplakin truncating variant. They postulated that different signaling pathways explain the different molecular signatures observed in LDAC and ARVC. ${ }^{22}$ We extended these previous findings using novel IHC markers to characterize selected protein distribution in LV myocardial tissue. Junction plakoglobin signal 
intensity was depressed or absent at the intercalated disks in the majority $(4 / 6 ; 67 \%)$ of our cases fulfilling ARVC criteria but signal intensity was normal at the intercalated disks in our two DCM cases, confirming the different molecular signatures of protein distribution patterns depending on the phenotype.

Immunofluorescence stainings for synapse-associated protein 97 and glycogen synthase kinase-3 beta confirmed the distinct molecular signature of PLN p.Arg14del cardiomyopathy in comparison to desmosomal ACM: synapse-associated protein 97 was previously shown to be consistently reduced in the ventricular myocardium of desmosomal ACM patients, an effect that was independent of specific causal mutation. ${ }^{13}$ We observed this in only one case (case 1). In 7/8 (88\%) cases, immunofluorescence for synapse-associated protein 97 showed a strong synapse-associated protein 97 signal at the sarcomeres, although this signal was gone from the intercalated disks. This pattern had previously been seen in myocardial samples from patients with end-stage ischemic, dilated, or hypertrophic cardiomyopathy. ${ }^{13}$ Immunofluorescence for glycogen synthase kinase-3 beta revealed that retained its cytoplasmic distribution and did not translocate to the intercalated disks in the same $7 / 8(88 \%)$ cases that showed a strong signal for synapse-associated protein 97 at the sarcomeres. Case 1 is the only one that showed junctional redistribution for glycogen synthase kinase-3 beta. In a desmosomal ACM cohort, all cases (20 out of 20 ) showed glycogen synthase kinase-3 beta junctional redistribution. ${ }^{14}$ Importantly, glycogen synthase kinase-3 beta downregulates SERCA2a leading to an inability to normalize cytosolic $\mathrm{Ca}^{2+}$ in diastole. ${ }^{23}$ Therefore, the disturbed SERCA2a/PLN interaction and subsequent calcium homeostasis in PLN p.Arg14del cardiomyopathy may play a role in the differential molecular signature observed. These new findings are in line with the phenotypical heterogeneity observed in PLN p.Arg14del cardiomyopathy.

Using targeted next-generation sequencing, we found that another gene variant (PV or VUS) was present in those cases with a diminished junction plakoglobin signal intensity at the intercalated disks $(4 / 8 ; 50 \%)$. The pathogenic variant in MYBPC3 (case 5), a sarcomeric gene, had been observed previously in another ARVC case but this may be a chance finding. ${ }^{24}$ In case 1 , where we observed an 
overall diminished synapse-associated protein 97 signal intensity and junctional redistribution of glycogen synthase kinase-3 beta, a VUS in the EMD-gene encoding emerin was detected in addition to the pathogenic PLN p.Arg14del variant (Table 1). Emerin is a nuclear envelope protein implicated in regulating muscle- and heart-specific gene expression and nuclear architecture. Due to the specific localization of emerin to desmosomes in the heart, ${ }^{25}$ this variant may play a role in the different distribution we observed in this case. Although it is important to note that the use of NGS techniques results in the identification of an increasing numbers of genetic variants and, consequently, of patients with complex genotypes, when interpreting the pathogenicity of genetic variants in ACM/DCM-related genes, we must be aware that putative pathogenic variants can also be present in healthy controls, albeit less frequently. ${ }^{26}$ Moreover, mutations outside well-known cardiomyopathy genes not analyzed here may also play a role. The interpretation of these data and identification of disease-causing variants and possible modifiers therefore remains a challenge.

To summarize and conclude, we have shown that PLN p.Arg14del cardiomyopathy has a distinct molecular signature in comparison to desmosomal ACM. It appears that the PLN p.Arg14del mutation does not by itself cause diminished junction plakoglobin at the intercalated disks, loss of synapse-associated protein 97 signal from the sarcomeres and junctional redistribution of glycogen synthase kinase-3 beta, as was shown in desmosomal ACM. Although limited by the small size of our cohort, this study substantiates the idea that additional genetic variants play a role in the phenotypical heterogeneity and in the differential protein distribution observed in PLN p.Arg14del cardiomyopathy. The highly arrhythmogenic overlapping phenotype and the pattern of biventricular subepicardial fibrosis and fatty infiltration supports the concept of ACM, i.e. biventricular subtypes. These distinct features characterize PLN p.Arg14del cardiomyopathy and provide further evidence for this disease as a biventricular disease entity within the ACM spectrum. 
Acknowledgements: We thank Kate Mc Intyre for carefully editing this manuscript.

Funding: This work was financially supported by a grant from the Fondation Leducq (CurePLaN) and the Netherlands Cardiovascular Research Initiative, an initiative supported by the Dutch Heart Foundation (The Hague, the Netherlands): CVON2012-10 PREDICT, CVON2014-40 DOSIS and CVON 2015-12 eDETECT projects. Wouter P. te Rijdt is supported by Young Talent Program (CVON PREDICT) grant 2017T001 from the Dutch Heart Foundation. The funding sources had no involvement in study design; in the collection, analysis and interpretation of data; in the writing of the report; and in the decision to submit the article for publication.

Conflicts of interest: None to declare.

Contributors: All authors have materially participated in the research and/or article preparation and have approved the final article. 


\section{References}

1 Watkins H, Ashrafian H, Redwood C: Inherited cardiomyopathies. N Engl J Med 2011; 364: 1643-1656.

2 Corrado D, Link MS, Calkins H: Arrhythmogenic Right Ventricular Cardiomyopathy. N Engl J Med 2017; 376: 61-72.

3 Te Rijdt WP, Jongbloed JD, de Boer RA et al: Clinical utility gene card for: arrhythmogenic right ventricular cardiomyopathy (ARVC). Eur J Hum Genet 2014; 22: 10.1038/ejhg.2013.124.

4 Lazzarini E, Jongbloed JD, Pilichou K et al: The ARVD/C genetic variants database: 2014 update. Hum Mutat 2015; 36: 403-410.

5 van der Zwaag PA, van Rijsingen IA, Asimaki A et al: Phospholamban R14del mutation in patients diagnosed with dilated cardiomyopathy or arrhythmogenic right ventricular cardiomyopathy: evidence supporting the concept of arrhythmogenic cardiomyopathy. Eur J Heart Fail 2012; 14: 1199-1207.

6 van der Zwaag PA, van Rijsingen IA, de Ruiter R et al: Recurrent and founder mutations in the NetherlandsPhospholamban p.Arg14del mutation causes arrhythmogenic cardiomyopathy. Neth Heart J 2013; 21: 286-293.

7 van Rijsingen IA, van der Zwaag PA, Groeneweg JA et al: Outcome in Phospholamban R14del Carriers: Results of a Large Multicentre Cohort Study. Circ Cardiovasc Genet 2014; Aug; 7: 455-65.

8 Gho JM, van Es R, Stathonikos N et al: High Resolution Systematic Digital Histological Quantification of Cardiac Fibrosis and Adipose Tissue in Phospholamban p.Arg14del Mutation Associated Cardiomyopathy. PLoS One 2014; 9: e94820.

9 Te Rijdt WP, van Tintelen JP, Vink A et al: Phospholamban p.Arg14del cardiomyopathy is characterized by phospholamban aggregates, aggresomes and autophagic degradation. Histopathology 2016; 69: 542-50.

10 Sepehrkhouy S, Gho JMIH, van Es R et al: Distinct fibrosis pattern in desmosomal and phospholamban mutation carriers in hereditary cardiomyopathies. Heart Rhythm 2017; 14: 1024-1032.

11 Mestroni L, Maisch B, McKenna WJ et al: Guidelines for the study of familial dilated cardiomyopathies. Collaborative Research Group of the European Human and Capital Mobility Project on Familial Dilated Cardiomyopathy. Eur Heart J 1999; 20: 93-102.

12 Marcus FI, McKenna WJ, Sherrill D et al: Diagnosis of arrhythmogenic right ventricular cardiomyopathy/dysplasia: proposed modification of the Task Force Criteria. Eur Heart J 2010; 31: 806-814.

13 Asimaki A, Kapoor S, Plovie E et al: Identification of a new modulator of the intercalated disc in a zebrafish model of arrhythmogenic cardiomyopathy. Sci Transl Med 2014; 6: 240 ra74.

14 Chelko SP, Asimaki A, Andersen P et al: Central role for GSK3beta in the pathogenesis of arrhythmogenic cardiomyopathy. JCI Insight 2016; 1: 10.1172/jci.insight.85923.

15 Sikkema-Raddatz B, Johansson LF, de Boer EN et al: Targeted next-generation sequencing can replace Sanger sequencing in clinical diagnostics. Hum Mutat 2013; 34: 1035-1042.

16 Richards S, Aziz N, Bale S et al: Standards and guidelines for the interpretation of sequence variants: a joint consensus recommendation of the American College of Medical Genetics and Genomics and the Association for Molecular Pathology. Genet Med 2015; 17: 405-424. 
17 Asimaki A, Tandri $\mathrm{H}$, Huang $\mathrm{H}$ et al: A new diagnostic test for arrhythmogenic right ventricular cardiomyopathy. N Engl J Med 2009; 360: 1075-1084.

18 Asimaki A, Protonotarios A, James CA et al: Characterizing the Molecular Pathology of Arrhythmogenic Cardiomyopathy in Patient Buccal Mucosa Cells. Circ Arrhythm Electrophysiol 2016; 9: e003688.

19 Groeneweg JA, van der Zwaag PA, Olde Nordkamp LR et al: Arrhythmogenic right ventricular dysplasia/cardiomyopathy according to revised 2010 task force criteria with inclusion of non-desmosomal phospholamban mutation carriers. Am J Cardiol 2013; 112: 1197-1206.

20 Te Rijdt WP, Ten Sande JN, Gorter TM et al: Myocardial fibrosis as an early feature in phospholamban p.Arg14del mutation carriers: phenotypic insights from cardiovascular magnetic resonance imaging. Eur Heart J Cardiovasc Imaging 2018; Apr 6. doi: 10.1093/ehjci/jey047. [Epub ahead of print].

21 Sen-Chowdhry S, Syrris P, Prasad SK et al: Left-dominant arrhythmogenic cardiomyopathy: an underrecognized clinical entity. J Am Coll Cardiol 2008; 52: 2175-2187.

22 Lopez-Ayala JM, Gomez-Milanes I, Sanchez Munoz JJ et al: Desmoplakin truncations and arrhythmogenic left ventricular cardiomyopathy: characterizing a phenotype. Europace 2014; 16: 1838-1846.

23 Michael A, Haq S, Chen X et al: Glycogen synthase kinase-3beta regulates growth, calcium homeostasis, and diastolic function in the heart. J Biol Chem 2004; 279: 21383-21393.

24 Murray B, Hoorntje ET, Te Riele ASJM et al: Identification of sarcomeric variants in probands with a clinical diagnosis of arrhythmogenic right ventricular cardiomyopathy (ARVC). J Cardiovasc Electrophysiol 2018; 29: 1004-1009.

25 Cartegni L, di Barletta MR, Barresi R et al: Heart-specific localization of emerin: new insights into EmeryDreifuss muscular dystrophy. Hum Mol Genet 1997; 6: 2257-2264.

26 Kapplinger JD, Landstrom AP, Salisbury BA et al: Distinguishing arrhythmogenic right ventricular cardiomyopathy/dysplasia-associated mutations from background genetic noise. J Am Coll Cardiol 2011; 57: 2317-2327. 


\section{Figures (2)}

Figure 1. Midventricular transverse myocardial slice of case 5 showing extensive fatty infiltration of the RV myocardium, especially at the outer anterior wall (outflow tract), and fibrofatty replacement of the LV myocardium, especially posterolaterally.

Figure 2. Immunofluorescence of left ventricular myocardial tissue (case 1, case 7, and control). Immunoreactive signals for desmoplakin and gap junction protein at intercalated discs are normal compared to control. $\mathrm{N}$-cadherin is used as a tissue quality control and is normal in all samples. The synapse-associated protein 97 signal is overall depressed in case 1 compared to control and case 7 . Glycogen synthase kinase-3 beta maintained its normal cytoplasmic distribution in case 7 (and control) but shows junctional redistribution in case 1. 